Traditional Medicine and Modern Medicine

Vol. 1, No. 1 (2018) 53-58

(C) Institutes of Integrative Medicine, Fudan University and

World Century Publishing Corporation

DOI: $10.1142 /$ S2575900018500040

\title{
Body mass index interaction with $S C N 10 A$ effects on cardiovascular autonomic neuropathy risk in a Chinese Han population
}

\author{
Guo-Zhuo Wang ${ }^{1}$, Zhi-Jun Liu ${ }^{1}$, Kai Xia ${ }^{1}$, Ke-Qin Zhang ${ }^{2}$ and Zi-Hui Tang ${ }^{1,2, *}$ \\ ${ }^{1}$ Department of Internal Medicine \\ Changde Hospital Affiliated to Hunan \\ University of Chinese Medicine, Hunan, P. R. China \\ ${ }^{2}$ Department of Endocrinology, Shanghai Tongji Hospital \\ Tongji University, Shanghai, P. R. China \\ *albert.tang@163.com
}

Received 6 July 2017; Accepted 1 December 2017; Published 14 February 2018

\begin{abstract}
Background: The aim of this study was to estimate the extent to which the interaction between SCN1OA (rs7375096) and body mass index (BMI) affects susceptibility to cardiovascular autonomic neuropathy (AN) in a Chinese Han sample.

Method: We conducted a large-scale, population-based study including 791 overweight patients and 1186 controls to analyze the interaction between SCN1OA and BMI with regard to CAN. All participants were genotyped for the presence of SCN10A (rs7375036) polymorphisms. Univariate and multiple linear regression analyses were employed to detect these relationships. The interaction between rs7375036 and BMI with regard to CAN susceptibility on an additive scale was estimated by using the relative excess risk due to interaction, the proportion attributable to interaction, and the synergy index.

Results: Univariate analyses found no significant association between rs7375036 single nucleotide polymorphism and CAN. After controlling for potential confounding factors, the multiple linear regression analysis showed a significant association between BMI and CAN $(P=0.021$, OR $=1.121)$. In addition, a significant positive interaction effect of BMI and rs7375036 on CAN was detected $(P=0.017)$.
\end{abstract}

Conclusion: Our findings suggest that BMI and SCN1OA (rs7375036) have interaction effects that influence the CAN.

Keywords: SCN10A; cardiovascular autonomic neuropathy; interaction; body mass index; Chinese Han population.

\section{Introduction}

Cardiovascular autonomic neuropathy (CAN) is a major complication of diabetes mellitus (DM), leading to increased morbidity and mortality in diabetic patients. CAN contributes to a high risk of cardiac arrhythmia, silent myocardial ischemia, and sudden death. ${ }^{1,2}$ It may occur when sympathetic and parasympathetic cardiovascular fibers are affected, which leads to neurohormonal compromise. The prevalence of CAN is estimated to be approximately $20-70 \%$, depending on the test used and the population investigated. ${ }^{1,3} \mathrm{CAN}$ also affects many other major segments of the general population, such as individuals with hypertension (HTN) and metabolic syndrome (MetS). ${ }^{4,5}$ In diabetic patients, MetS has been reported to be associated with cardiovascular autonomic (CA) function indices or impaired CA function. .,

People classified as overweight or obese exhibit increased risk of cardiovascular disease compared to those of normal weight. ${ }^{7}$ A meta-analysis of five longitudinal cohort studies demonstrated that overweight or obese diabetic patients had a twofold greater relative risk of mortality than normal-weight patients. ${ }^{8}$ Obesity is often considered as an important component of MetS and an independent risk factor for DM in Chinese populations. ${ }^{9}$ Our previous studies indicated that

\footnotetext{
*Corresponding author.
} 
MetS was associated with CAN in the general Chinese population. ${ }^{10,11}$ However, the documented association of obesity or overweight with CAN in several ethnic populations ${ }^{12,13}$ has not been replicated in the general Chinese population.

Several recent genome-wide association studies (GWAS) ${ }^{14,15}$ have linked SCN10A to the PR interval and QRS duration. The SCN1OA gene encodes the neuronal sodium channel isoform, NaV1.8. The single nucleotide polymorphism (SNP) of rs6795970 within the SCN10A gene carries a higher risk for heart blockages associated with cardiac conduction abnormalities in patients with hypertrophic cardiomyopathy. ${ }^{16}$ Previous studies provided evidence for gene-obesity interactions in complex human diseases. ${ }^{17,18}$ It is evident that obesity/overweight is associated with CAN, and SCN1OA is associated with cardiac electrophysiology. Our previous studies demonstrated that MetS interacted with estrogen receptor 1 (ESRI) and DM interacted with SCN10A on CAN, ${ }^{19,20}$ respectively. Based on our previous studies, we hypothesize that obesity/overweight may modify the association between $S C N 1 O A$ and CAN via the interactions ofSCN1OA and BMI with CAN. The aim of this study was to explore the risk factors for CAN and the interaction effects of $S C N 10 \mathrm{~A}$ and body mass index (BMI) on CAN susceptibility in a large-scale clinical setting.

\section{Methods}

\section{Study population}

As described earlier, ${ }^{19-21}$ this study is based on a communitybased dataset in Chinese population. We recruited participants from rural and urban communities in Shanghai, and those aged 30-80 years with undiagnosed CAN were included. Written consent was obtained from all participants before the study. This study was approved by the Ethics Committee of Shanghai Tongji Hospital of Tongji Univeristy, Shanghai, China. The methods were carried out in accordance with the approved guidelines. Our study invited a total of 3012 subjects to a screening visit between 2011 and 2013. The exclusion criteria were detailed in our earlier study ${ }^{19-21}$; some subjects were excluded to eliminate potential confounding factors that may have influenced their CA function.

\section{Measurements}

As described in our earlier study, ${ }^{19-21}$ the participants were interviewed to document their medical histories, medications, and smoking habits. Laboratory assessments of cardiovascular disease risk factors were completed along with standardized examinations for heart rate variability (HRV). The evaluation of demographic information, glucose profiles, lipid profiles, renal function, HRV indices, and medical histories was described previously. ${ }^{19,20}$ The definitions of BMI, HTN, DM, and MetS were provided in our previous study. ${ }^{19,20}$ The genotyping for SNP of rs7375036 was detailed earlier. ${ }^{20}$ Briefly, the genomic DNA was isolated from whole blood by proteinase $\mathrm{K}$ digestion followed by phenol-chloroform extraction. The rs7375036 in 2092 Chinese Han participants was genotyped. Of these subjects, 1977 participants with complete clinical and genotype data were available for data analysis in this study.

As mentioned in our earlier study, ${ }^{19,20}$ short-term HRV was applied to evaluate CA function. HRV was measured non-invasively by power spectral analysis. CAN was diagnosed based on at least two abnormal CA reflex test results based on short-term HRV tests. ${ }^{20,22,23}$ A total of 1977 participants with complete clinical and genotype data were available for data analysis in this study.

\section{Statistical analysis}

Differences in variables between obesity and normal BMI participants were determined with an unpaired $t$-test. Between groups, differences in properties were assessed with the $\chi^{2}$ analysis. Multivariable logistic regression (MLR) was carried out to control potential confounders to determine the independent associations of variables with CAN. Interaction analyses were detailed in our earlier study, ${ }^{19,20}$ MLR was conducted to include two main variables and its interaction item to evaluate the interaction effect. The odds ratio (OR) with a 95\% confidence interval (CI) was calculated for the relative risk of BMI and SNP with regard to CAN. Three parameters, relative excess risk due to interaction (RERI), proportion attributable to interaction (AP), and synergy index $(S)$, were used to estimate measures of interaction on an additive scale. The results were analyzed using the Statistical Package for Social Sciences for Windows version 16.0 (SPSS, Chicago, IL, USA). Tests were two-sided, and a $P$-value of $<0.05$ was considered significant.

\section{Results}

\section{Baseline characteristics of participants}

The baseline clinical characteristics of the 1977 participants were described earlier, ${ }^{19-21}$ and are listed in Table 1. Participants with excess weight had significantly higher weights than those with normal BMI $(71.84 \mathrm{~kg}$ versus $57.70 \mathrm{~kg}$, $P<0.01)$. The prevalence rates of HTN, DM, MetS, and CAN were also significantly higher in participants with excess weight $(P<0.001$ for all). Systolic blood pressure (SBP), diastolic blood pressure (DBP), glucose levels, lipid levels, and heart rate were higher in participants with excess weight compared to those with normal BMI, while there were 
Table 1. The baseline characteristic of individuals.

\begin{tabular}{|c|c|c|c|c|}
\hline Variable & Normal BMI & Excess weight & Total sample & $P$-value* \\
\hline \multicolumn{5}{|c|}{ Demographic information } \\
\hline$N$ & 1186 & 791 & 1977 & - \\
\hline Age years & $59.99 \pm 8.63$ & $61.27 \pm 8.77$ & $60.5 \pm 8.71$ & $<0.001$ \\
\hline Gender male, $\%$ & $368(31.03 \%)$ & $299(37.8 \%)$ & $667(33.74 \%)$ & $<0.001$ \\
\hline Height $\mathrm{cm}$ & $161.45 \pm 7.83$ & $161.54 \pm 7.87$ & $161.49 \pm 7.85$ & 0.729 \\
\hline Weight kg & $57.7 \pm 7.7$ & $71.84 \pm 8.77$ & $63.3 \pm 10.68$ & $<0.001$ \\
\hline SBP mmHg & $124.57 \pm 18.19$ & $132.46 \pm 18.77$ & $127.71 \pm 18.82$ & $<0.001$ \\
\hline DBP $\mathrm{mmHg}$ & $78.14 \pm 9.33$ & $82.28 \pm 9.7$ & $79.79 \pm 9.69$ & $<0.001$ \\
\hline \multicolumn{5}{|l|}{ HRV indices } \\
\hline HR bpm & $72.2 \pm 10.32$ & $72.99 \pm 9.89$ & $72.52 \pm 10.16$ & 0.018 \\
\hline $\mathrm{TP} \mathrm{ms}^{2}$ & $894.05 \pm 724.14$ & $857.28 \pm 685.73$ & $879.34 \pm 709.16$ & 0.11 \\
\hline $\mathrm{LF} \mathrm{ms}^{2}$ & $194.82 \pm 205.7$ & $188.49 \pm 214.59$ & $192.29 \pm 209.3$ & 0.351 \\
\hline $\mathrm{HF} \mathrm{ms}^{2}$ & $185.64 \pm 234.32$ & $183.27 \pm 201.17$ & $184.69 \pm 221.63$ & 0.743 \\
\hline LFHF & $1.75 \pm 2.04$ & $1.62 \pm 1.88$ & $1.7 \pm 1.98$ & 0.051 \\
\hline \multicolumn{5}{|l|}{ Laboratory assays } \\
\hline FPG mmol/L & $5.34 \pm 1.71$ & $5.69 \pm 1.86$ & $5.48 \pm 1.78$ & $<0.001$ \\
\hline $\mathrm{PBG} \mathrm{mmol} / \mathrm{L}$ & $7.17 \pm 3.45$ & $8.26 \pm 3.66$ & $7.6 \pm 3.58$ & $<0.001$ \\
\hline FINS uml/L & $6.18 \pm 9.19$ & $8.87 \pm 15.44$ & $7.26 \pm 12.16$ & $<0.001$ \\
\hline $\mathrm{TC} \mathrm{mmol} / \mathrm{L}$ & $5.34 \pm 0.99$ & $5.28 \pm 1.02$ & $5.32 \pm 1$ & 0.049 \\
\hline TG mmol/L & $1.59 \pm 0.86$ & $1.89 \pm 1.12$ & $1.71 \pm 0.99$ & $<0.001$ \\
\hline $\mathrm{HDL} \mathrm{mmol} / \mathrm{L}$ & $1.41 \pm 0.34$ & $1.27 \pm 0.28$ & $1.36 \pm 0.33$ & $<0.001$ \\
\hline $\mathrm{LDL} \mathrm{mmol} / \mathrm{L}$ & $3.17 \pm 0.78$ & $3.22 \pm 0.77$ & $3.19 \pm 0.78$ & 0.027 \\
\hline $\mathrm{SCr} \mu \mathrm{mol} / \mathrm{L}$ & $77.18 \pm 29.37$ & $78.18 \pm 21.77$ & $77.58 \pm 26.59$ & 0.249 \\
\hline $\mathrm{UA} \mu \mathrm{mol} / \mathrm{L}$ & $269.08 \pm 81$ & $297.66 \pm 85.93$ & $280.51 \pm 84.17$ & $<0.001$ \\
\hline \multicolumn{5}{|l|}{ Medical history } \\
\hline Smoking yes,\% & $163(13.74 \%)$ & $141(17.83 \%)$ & $304(15.38 \%)$ & $<0.001$ \\
\hline HTN yes, $\%$ & $455(38.36 \%)$ & $473(59.8 \%)$ & $928(46.94 \%)$ & $<0.001$ \\
\hline DM yes, $\%$ & $192(16.2 \%)$ & $227(28.7 \%)$ & $419(21.2 \%)$ & $<0.001$ \\
\hline MetS yes, $\%$ & $308(25.97 \%)$ & $475(60.05 \%)$ & $783(39.61 \%)$ & $<0.001$ \\
\hline CAN yes, \% & $196(16.53 \%)$ & $167(21.11 \%)$ & $363(18.36 \%)$ & $<0.001$ \\
\hline \multicolumn{5}{|l|}{ SNP } \\
\hline rs7375036 (C) & $20(1.70 \%)$ & $17(2.09 \%)$ & $37(1.96 \%)$ & 0.367 \\
\hline
\end{tabular}

Notes: *Difference analysis between non-MetS and MetS groups; SBP-systolic blood pressure, DBP-diastolic blood pressure, FPG-fasting plasma glucose, PBG-plasma blood glucose, FINSfasting blood insulin, TC-serum total cholesterol, TG-triglyceride, UA-uric acid, HDL-high-density lipoprotein cholesterol, LDL-low-density lipoprotein cholesterol, SCr-serum creatinine, UA-uric acid, HR-heart rate, TP-total power of variance, LF-low frequency, HF-high frequency, HTN-hypertension, MetS-metabolic syndrome, DM-diabetes mellitus, CAN-cardiovascular autonomic neuropathy, SNPsingle nucleotide polymorphism.

lower high-density lipoprotein (HDL) cholesterol levels in participants with excess weight. As mentioned in our previous study, ${ }^{20}$ the minor allele $(C)$ frequencies of rs 7375036 within the SCN1OA gene were $1.70 \%$ and $2.09 \%$ in participants with excess weight and those with normal BMI, respectively.

\section{Univariate and multiple variable analysis for diabetes}

Univariate logistic regression models were developed to estimate the associations between various clinical factors and CAN. As described earlier, ${ }^{20,21}$ the univariate analyses revealed that age, BMI, SBP, DBP, glucose level, triglyceride (TG), and medical history were significantly associated with CAN $(P<0.05$ for all); however, there were no significant associations between rs7375036 SNP and CAN $(P=0.627)$.
The MLR that included BMI and rs7375036 SNP, after controlling for the potential confounding factors of age, gender, blood pressure, glucose level, TG, and medical history, indicated a significant association between BMI and CAN $(P=0.021$, OR $=1.121,95 \%$ CI: $1.018-1.234$, Table 2). However, there was no significant association between rs7375036 SNP and CAN $(P=0.699)$.

\section{BMI and SCN10A interaction analysis for CAN}

MLR models were developed to include the two main effect variables of BMI and rs7375036 SNP and their interaction (BMI*rs7375036). The interaction item among them was detected in the MLR model after adjustment for the relevant potential confounders of age, gender, blood pressure, glucose level, TG, and medical history $(P=0.017)$ (Table 3 and Fig. 1). In participants with normal BMI, the CAN 
Table 2. Multiple variable analysis for BMI and rs7375036 for CAN.

\begin{tabular}{lccccc}
\hline Variables & $\beta$ & $\mathrm{SE}$ & $P$-value & $\mathrm{OR}$ & $95.0 \% \mathrm{CI}$ \\
\hline BMI & 0.114 & 0.049 & 0.021 & 1.121 & $1.018-1.234$ \\
rs7375036 & 0.116 & 0.301 & 0.699 & 1.124 & $0.623-2.028$ \\
\hline
\end{tabular}

Notes: DM-diabetes mellitus, BMI-body mass index, multiple variables logistic model adjusted for age, gender, blood pressure, laboratory assays, and medical history.

Table 3. The interaction analysis of BMI and SNPs for CAN.

\begin{tabular}{lrcccc}
\hline Variables & $\beta$ & SE & $P$-value & OR & $95.0 \%$ CI \\
\hline BMI & 0.266 & 0.084 & 0.002 & 1.304 & $1.106-1.537$ \\
rs7375036 & -0.006 & 0.013 & 0.133 & 0.994 & $0.878-1.127$ \\
BMI by rs7375036 & 1.687 & 0.706 & 0.017 & 5.404 & $1.355-21.558$ \\
RERI & & 5.706 & & $3.781-8.613$ \\
AP & \multicolumn{5}{c}{0.815} \\
& 20.149 & & $-0.185-1.814$ \\
$S$ & & & $9.567-32.433$ \\
\hline
\end{tabular}

Notes: DM-diabetes mellitus, RERI-relative excess risk due to interaction, AP-proportion attributable to interaction, $S$-synergy index.

prevalence was lower in those with minor allele $C$ and those with allele $T(10.21 \%$ and $15.54 \%$, respectively). In contrast, in participants with excess weight, the CAN prevalence was significantly higher in those with minor allele $C$ compared to those with allele $T(37.29 \%$ versus $19.79 \%)$. The interaction effect was estimated $\left(\mathrm{OR}_{\mathrm{Int}}=5.404,95 \% \mathrm{CI}: 1.355-21.558\right)$ as was the interaction on an additive scale (RERI $=5.706$, 95\%CI: 3.781-8.613; AP $=0.815$, 95\%CI: $-0.185-1.814$; $S=20.149,95 \%$ CI: 9.567-32.433)

\section{Discussion}

We conducted a large-scale, community-based, cross-sectional study to evaluate the interaction effect of BMI and SCN1OA on

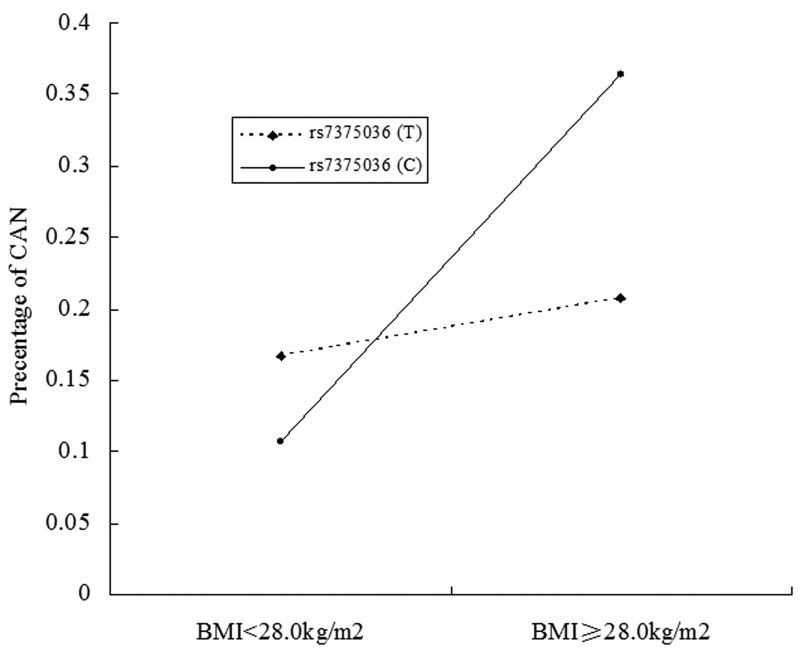

Figure 1. Interaction analysis of SCN10A (7375036) and BMI for CAN. Interaction of BMI and SAN10A (rs7375036), BMI*rs7375036 $\mathrm{OR}_{\mathrm{GEI}}=5.404,95 \%$ CI: $1.355-21.558, P=0.017$.
CAN in a large case-control sample of Chinese individuals. To the best of our knowledge, this is the first investigation with an interaction analysis of CAN with regard to BMI and SCN1OA in a Chinese population. The short-term HRV was measured non-invasively by a power spectral analysis to evaluate CA function. This test is suitable for population-based studies. The prevalence of HTN, MetS, and CAN in the general population was similar to the previous studies. Our sample was a good representation across the country, so our findings might work well outside of the studied areas in China.

The present study reconfirmed the fact that BMI is significantly associated with CAN. Univariate and multiple variable analyses provided evidence to support this finding $(P<0.001)$, and the results were consistent with the previous reports. ${ }^{12,13}$ However, no associations between SCN1OA and CAN were found in the present study $(P=0.699)$. The functional presence of $S C N 10 \mathrm{~A} / \mathrm{NaV} 1.8$ in intracardiac neurons indicates a novel role for this neuronal sodium channel in the regulation of cardiac electric activity; however, there is no evidence suggesting that SCN10A polymorphisms are associated with an increased relative risk of CAN. In neural tissues, the sodium channel isoform NaV1.8 (encoded by the SCN1OA gene) is highly expressed in small- and medium-diameter nociceptive sensory neurons of the dorsal root ganglia and cranial sensory ganglia. ${ }^{24}$ There is little evidence to demonstrate SCN1OA (rs7375096) to be an independent risk factor for CAN. This may be attributed to the limited number of participants, which resulted in insufficient statistical power to detect a slight effect of the common polymorphism in SCN1OA on CAN susceptibility. Therefore, it is necessary to determine the association between this SCN10A genetic variant and CAN in a larger sample size. Another possible explanation for this puzzling phenomenon is the diverse ethnic/regional backgrounds of the participants as the findings might vary by population because of underlying, unobserved genetic variation.

These interesting findings showed that there was a significant interaction effect of BMI and SCN1OA on CAN in a Chinese population. Our study is the first analysis of the interaction of BMI and SCN1OA variants on CAN susceptibility. Among several factors, autonomic dysregulation has been advocated as a causal link between unhealthy lifestyles, such as overeating and sedentariness, and metabolic abnormalities, as modeled by MetS. ${ }^{25}$ Considering that lifestyle changes can modify the risk of CAN, the likely effect of metabolic quantitative traits on CAN susceptibility needs to be addressed as well as their interactions with various gene variants. NaV1.8 function has mostly been associated with pain perception, ${ }^{26}$ but several GWAS have also linked SCN10A to PR interval and QRS duration on ECG. ${ }^{14,15,27}$ The association between the SCN1OA locus and atrial fibrillation has consistently been reported. ${ }^{14}$ These findings 
indicate that SCN1OA might be a potential genetic marker for CAN. In the present study, the interaction of rs7375096 ( $C$ allele) and BMI conferred a high risk for CAN. Additionally, our previous study demonstrated that DM interacts SCN10A to affect CAN in this sample. ${ }^{20}$ There findings offered evidence what SCN1OA associated with CAN was modified by environmental factors such as metabolic factors.

Autonomic dysfunction depends not only on autonomic nervous system function, but also on the integrity of the CA reflex loops. CAN has been attributed to increased tunica intima or media thickness at the site of the baroreceptors, reduced vascular distensibility, endothelial dysfunction, and impaired cardiac contractility, which also contributes to CA dysfunction. ${ }^{28}$ It has already been demonstrated that lifestyle modifications and improvements in HTN and/or weight control can prevent the progression of CAN. ${ }^{29}$ In this study, we focused on evaluating the relationships of SCN1OA variants and $\mathrm{BMI}$ to $\mathrm{CAN}$. It is noteworthy that the risk imparted by the minor $C$ allele was higher than that by the $T$ allele in overweight subjects, thus prompting speculation about a possible interaction between SCN10A (rs7375096) and BMI in determining overall CAN risk. However, the underlying mechanism is still unknown.

This study had several limitations. Firstly, the participants were all recruited from Shanghai, so they may not have been representative of China as a whole. Additionally, this study did not cover age groups outside of 30-80 years, so it is less practical for younger or elderly populations. Furthermore, the present study was based on a cross-sectional design, so it was not possible to detect individual changes in autonomic regulation related to lifestyle changes, or to perform a comparative simultaneous evaluation of interventions on BMI and CA function. Finally, it is important to confirm these findings with additional investigations using larger samples to systematically evaluate the likely interaction effect of SCN10A variants and BMI on CAN risk. The synergistic results of this study need to be verified by future follow-up studies.

\section{Conclusion}

Our findings suggested that BMI is independently and significantly associated with CAN, and offered evidence of an interactive effect of SCN10A (rs7375096) and BMI on CAN. The environmental factor of BMI may modify the SCN10A gene to associate it with the CAN. A functional study, such as gene-targeting in mice, is needed to clarify the role of SCN1OA as a whole.

\section{Conflict of Interest}

The authors have no conflict of interest to declare.

\section{Acknowledgments}

We thank the grant from Shanghai Tongji Hosptial, Tongji University to support the study.

\section{References}

1. Spallone V, Ziegler D, Freeman R, et al. Cardiovascular autonomic neuropathy in diabetes: Clinical impact, assessment, diagnosis, and management. Diabetes Metab Res Rev 2011;27:639-53.

2. Pop-Busui R, Evans GW, Gerstein HC, et al. Effects of cardiac autonomic dysfunction on mortality risk in the Action to Control Cardiovascular Risk in Diabetes (ACCORD) trial. Diabetes Care 2010;33:1578-84.

3. Fleischer J. Diabetic autonomic imbalance and glycemic variability. J Diabetes Sci Technol 2012;6:1207-15.

4. Hazari MA, Khan RT, Reddy BR, Hassan MA. Cardiovascular autonomic dysfunction in type 2 diabetes mellitus and essential hypertension in a South Indian population. Neurosciences 2012;17:173-5.

5. Garruti G, Giampetruzzi F, Vita MG, et al. Links between metabolic syndrome and cardiovascular autonomic dysfunction. Exp Diabetes Res 2012;2012:615835.

6. Chang CJ, Yang YC, Lu FH, et al. Altered cardiac autonomic function may precede insulin resistance in metabolic syndrome. Am J Med 2010;123:432-8.

7. Gong CD, Wu QL, Chen Z, Zhang D, Zhao ZY, Peng YM. Glycolipid metabolic status of overweight/obese adolescents aged 9- to 15-year-old and the BMI-SDS/BMI cut-off value of predicting dyslipidemiain boys, Shanghai, China: A crosssectional study. Lipids Health Dis 2013;12:129.

8. Carnethon MR, De Chavez PJ, Biggs ML, et al. Association of weight status with mortality in adults with incident diabetes. JAMA 2012;308:581-90.

9. Xin Z, Liu C, Niu WY, et al. Identifying obesity indicators which best correlate with type 2 diabetes in a Chinese population. BMC Public Health 2012;12:732.

10. Zhang J, Liu NJ, Zhang Y, Yang H, Tang Z. The association and interaction analysis of metabolic syndrome and chronic kidney disease on cardiovascular autonomic neuropathy in the general Chinese population. Int J Clin Exp Med 2015;8:9649-57.

11. Lu Y, Tang ZH, Zeng F, Li Y, Zhou L. The association and predictive value analysis of metabolic syndrome combined with resting heart rate on cardiovascular autonomic neuropathy in the general Chinese population. Diabetol Metab Syndr 2013;5:73.

12. Sant Anna Junior M, Carneiro JR, Carvalhal RF, et al. Cardiovascular autonomic dysfunction in patients with morbid obesity. Arq Bras Cardiol 2015;105:580-7.

13. Criado JR, Gilder DA, Kalafut MA, Ehlers CL. Obesity in American Indian and Mexican American men and women: Associations with blood pressure and cardiovascular autonomic control. Cardiovasc Psychiatry Neurol 2013;2013:680687.

14. Pfeufer A, van Noord C, Marciante KD, et al. Genome-wide association study of PR interval. Nat Genet 2010;42:153-9.

15. Holm H, Gudbjartsson DF, Arnar DO et al. Several common variants modulate heart rate, PR interval and QRS duration. Nat Genet 2010;42:117-22.

16. Iio C, Ogimoto A, Nagai T, et al. Association between genetic variation in the SCN10A gene and cardiac conduction 
abnormalities in patients with hypertrophic cardiomyopathy. Int Heart J 2015;56:421-7.

17. Lamina C, Forer L, Schonherr S et al. Evaluation of gene-obesity interaction effects on cholesterol levels: A genetic predisposition score on HDL-cholesterol is modified by obesity. Atherosclerosis 2012;225:363-9.

18. Wang X, Ding X, Su S et al. Heritability of insulin sensitivity and lipid profile depend on BMI: Evidence for gene-obesity interaction. Diabetologia 2009;52:2578-84.

19. Zeng F, Zhou L, Tang Z. A study for association and interaction analysis to metabolic syndrome and the ESR1 gene on cardiovascular autonomic neuropathy in a Chinese Han population. Diabetol Metab Syndr 2016;8:42.

20. Lv Y, Zhou L, Tang Z, Dong J. Association and interaction analysis of diabetes mellitus and SCN10A for cardiovascular autonomic neuropathy in a Chinese population. Postgrad Med J 2016.

21. Liu J, Tang ZH, Zeng F, Li Z, Zhou L. Artificial neural network models for prediction of cardiovascular autonomic dysfunction in general Chinese population. BMC Med Inform Decis Mak 2013;13:80.

22. Zeng F, Tang ZH, Li Z, Yu X, Zhou L. Normative reference of short-term heart rate variability and estimation of cardiovascular autonomic neuropathy prevalence in Chinese people. J Endocrinol Invest 2014;37:385-91.
23. Tang ZH, Wang L, Zeng F, et al. Bayesian estimation of cardiovascular autonomic neuropathy diagnostic test based on short-term heart rate variability without a gold standard. BMJ Open 2014;4:e005096.

24. Benn SC, Costigan M, Tate S, Fitzgerald M, Woolf CJ. Developmental expression of the TTX-resistant voltage-gated sodium channels Nav1.8 (SNS) and Nav1.9 (SNS2) in primary sensory neurons. J Neurosci 2001;21:6077-85.

25. Cerutti F, Rabbia F, Rabbone, I et al. Impairment of cardiovascular autonomic pattern in obese adolescents with Type 2 diabetes mellitus. J Endocrinol Invest 2010;33:539-43.

26. Liu M, Wood JN. The roles of sodium channels in nociception: Implications for mechanisms of neuropathic pain. Pain Med 2011;12(3):S93-9.

27. Chambers JC, Zhao J, Terracciano CM, et al. Genetic variation in SCN10A influences cardiac conduction. Nat Genet 2010;42:149-52.

28. Lefrandt JD, Smit AJ, Zeebregts CJ, Gans RO, Hoogenberg $\mathrm{KH}$. Autonomic dysfunction in diabetes: A consequence of cardiovascular damage. Curr Diabetes Rev 2010;6:348-58.

29. Tahrani AA, Dubb K, Raymond NT, et al. Cardiac autonomic neuropathy predicts renal function decline in patients with type 2 diabetes: A cohort study. Diabetologia 2014;57:1249-56. 